\title{
An objective, markerless videosystem for staging facial palsy
}

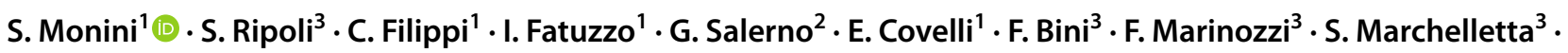 \\ G. Manni ${ }^{3} \cdot$ M. Barbara ${ }^{1}$ (1)
}

Received: 20 November 2020 / Accepted: 4 February 2021 / Published online: 15 March 2021

(c) The Author(s) 2021

\begin{abstract}
Purpose To propose a new objective, video recording method for the classification of unilateral peripheral facial palsy (UPFP) that relies on mathematical algorithms allowing the software to recognize numerical points on the two sides of the face surface that would be indicative of facial nerve impairment without positioning of markers on the face.

Methods Patients with UPFP of different House-Brackmann (HB) degrees ranging from II to V were evaluated after video recording during two selected facial movements (forehead frowning and smiling) using a software trained to recognize the face points as numbers. Numerical parameters in millimeters were obtained as indicative values of the shifting of the face points, of the shift differences of the two face sides and the shifting ratio between the healthy (denominator) and the affected side (numerator), i.e., the asymmetry index for the two movements.

Results For each HB grade, specific asymmetry index ranges were identified with a positive correlation for shift differences and negative correlation for asymmetry indexes.

Conclusions The use of the present objective system enabled the identification of numerical ranges of asymmetry between the healthy and the affected side that were consistent with the outcome from the subjective methods currently in use.
\end{abstract}

Keywords Facial palsy $\cdot$ Subjective grading system $\cdot$ Objective grading system $\cdot$ Videorecording $\cdot$ Smartphone $\cdot$ Machine learning

\section{Introduction}

Objective systems for grading unilateral peripheral facial palsy (UPFP) started to be proposed with the aim to overcome the several flaws revealed by the traditional, subjective methods. If it is true that these latter methods, such as the House-Brackmann or HB [1] or the Sunnybrook or SBGS [2] method, fail to highlight some important features of a facial impairment or its sequel, it is also true that the use of software-based systems could at times be difficult to use and, although objective and accurate [3-9], could also fail to

M. Barbara

Maurizio.barbara@uniroma1.it

1 ENT Clinic, NESMOS Department, Faculty of Medicine and Psychology, Sapienza University, Rome, Italy

2 Laboratory Unit, Sant'Andrea University Hospital, Via di Grottarossa 1035, 00189 Rome, Italy

3 Department of Mechanical and Aerospace Engineering, Faculty of Civil and Industrial Engineering, Sapienza University Rome, Rome, Italy consider all the aspects of the facial disfiguration, both with two- and three-dimensional methodologies [10-14]. In this regard, systems sensitive to global or partial changes of the face or to the presence of synkineses or secondary defects changes have still to be introduced in clinical practice.

In an attempt to elaborate an objective method for this purpose, an automatic software-based system has previously been reported and validated for the evaluation of UPFP via the analysis of shifting of markers preliminarily placed on specific face regions $[15,16]$. Although the system was appropriate for most UPFP cases and also consistent with the outcome from the subjective HB grading system, it is likely that marker placement may represent a biasing variable that is conditioned by the examiner's experience and by the different physiognomic characteristics of some affected individuals presenting with a poorly defined eyelid region, especially during closure and opening of the eyes.

The purpose of the present study was to elaborate a video recording automatic system for grading patients with UPFP without using facial markers. In this regard, the study group was composed of subjects who were previously classified 
and validated with another objective video recording system $[15,16]$.

\section{Materials and methods}

Forty subjects affected by UPFP were recruited for the present study. The subjects were consecutively included in each corresponding HB stage, from II to V, with ten subjects per group. All these subjects were previously assessed by a marker-based system [15] that provided data significantly correspondent to those obtained via both HB and Sunnybrook grading systems [16].

The main steps of the marker-based procedure were as follows:

- Placement of markers in both sides of the face at the levels of the upper, medium and lower sectors;

- Capture the frontal view of the subjects' face via video using a smartphone camera with flash on to enhance marker reflectivity.
Video recording lasted $15 / 20$ s for each patient. The patient was first asked to remain still and then to perform five common facial expression, including frown the forehead, mild eye closure, strong eye closure, smile and kiss, returning to the resting position after each movement.

In the present study, a new automatic system was applied to the same group of patients, enabling the tracing of face points without positioning of the markers. This procedure starts with the individuation of the facial points using a machine-learning algorithm that allows the automatic individuation of the face points [17]. By doing so, a ROI (region of interest) is added to the algorithm. This algorithm is based on a Histogram of Oriented Gradients (HOG) combined with a vector machine of support to obtain a plane containing the patient's face. Subsequently, using an algorithm based on "1 ms face alignment" with an ensemble of regression trees [18], 68 facial points were identified (Fig. 1). The identification of these 68 points was derived by decisional trees trained on one thousand face images in movement manually individuated. Therefore, the algorithm individuated the 68 points on the ROI, yielding the coordinates in pixels with a
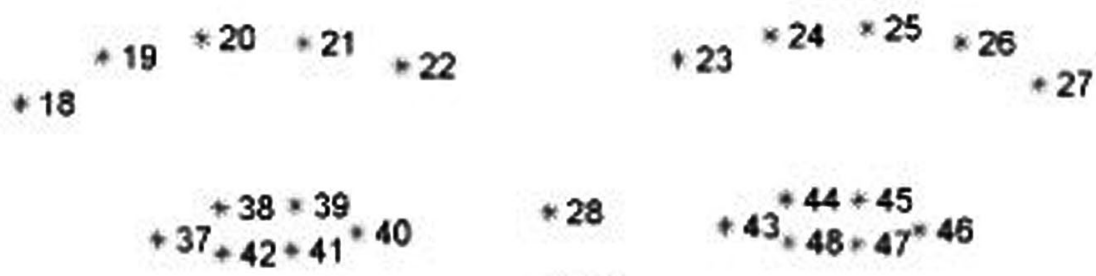

$+29$

$\cdot 1$

$* 30$

$-2$

$+31$

$+3$

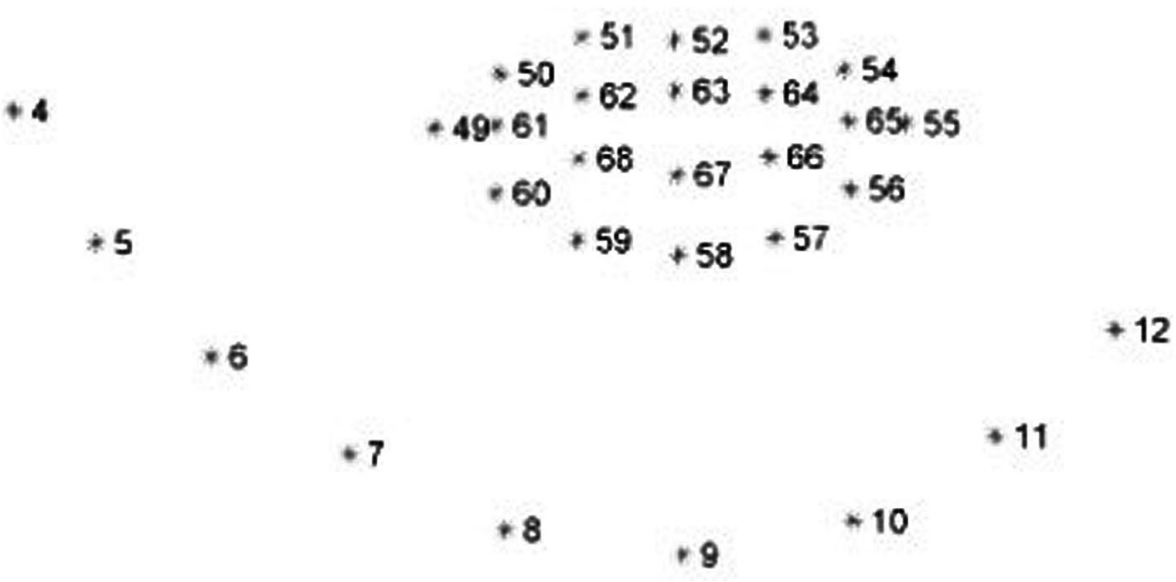

Fig. 1 Numbers related to specific points of anatomical facial structures individuated by a machine learning algorithm [18] 
68-line matrix (one for each point) and 2 columns (one for the $x$-axis, the other one for the $y$-axis). Before the elaboration, the measurements were converted from pixels into millimeters, considering $5 \mathrm{~mm}$ for each marker given that the markers were obtained by an automatic sheet-punching machine, and the dimensions of the markers in pixels on the image, based on the following formula:

$t=\frac{f[m m]}{f[p x]}$.

The following points were considered significant (Fig. 2):

- 20 and 25 for the eyebrow region

- 38 and 45 for the upper eyelid rim

- 42 and 47 for the lower eyelid rim

- 49 and 55 for the mouth corner

- 63 center of the mouth

$\left(\begin{array}{cc}x_{1} & y_{1} \\ \cdot & \cdot \\ x_{68} & y_{68}\end{array}\right)$.

Afterward, the video elaboration began with the window "import video" that uploaded all the videos, selecting the frames of interest. Once obtained, these data were reported in graphs using the module "matplotlib" and memorized on a.csv (comma-separated values) file accessible by Excel.

By doing so, the software delivered graphs and numerical data for each ROI on a specific frame window. It was, therefore, possible to perform a quantitative assessment of UPFP by evaluating the eventual shift of the selected point for the healthy side in comparison of that of the affected side, in patients who were already classified and validated in a previous study [16].

Two face movements (forehead frowning and smiling) were analysed in the normal and in the affected side, comparing the marker and the markerless methods.

Initially, the maximum distances of the eyebrow shift from the eyelid in the forehead frowning and the shift of the mouth corner from the mouth center during smiling were calculated in both sides of the face. To provide reliable quantitative values of the real shift for each side, the mean distance at rest was subtracted from the maximum distance during each movement.

The second step involved calculating the shift differences between the healthy and the affected side for each movement in patients with different HB grades. As result, mean partial and total values of specific shift differences for each HB grade were obtained.

The last step involved the assessment of the shifting ratio between the affected and the healthy side, using the former as a nominator and the latter as a denominator, to derive the asymmetry index between the two face sides. Similarly, for the asymmetry index both for partial and total scores of the two face movements considered, specific ranges for each $\mathrm{HB}$ group of patients were drawn.

\section{Statistical analysis}

Continuous data were summarized by mean and standard deviations (SD). The comparison of the shift difference and asymmetry index between HBII, HBIII, HBIV and HBV was evaluated using a one-way ANOVA test. The $p$ values are two sided, and a $p$ value $\leq 0.05$ was considered statistically

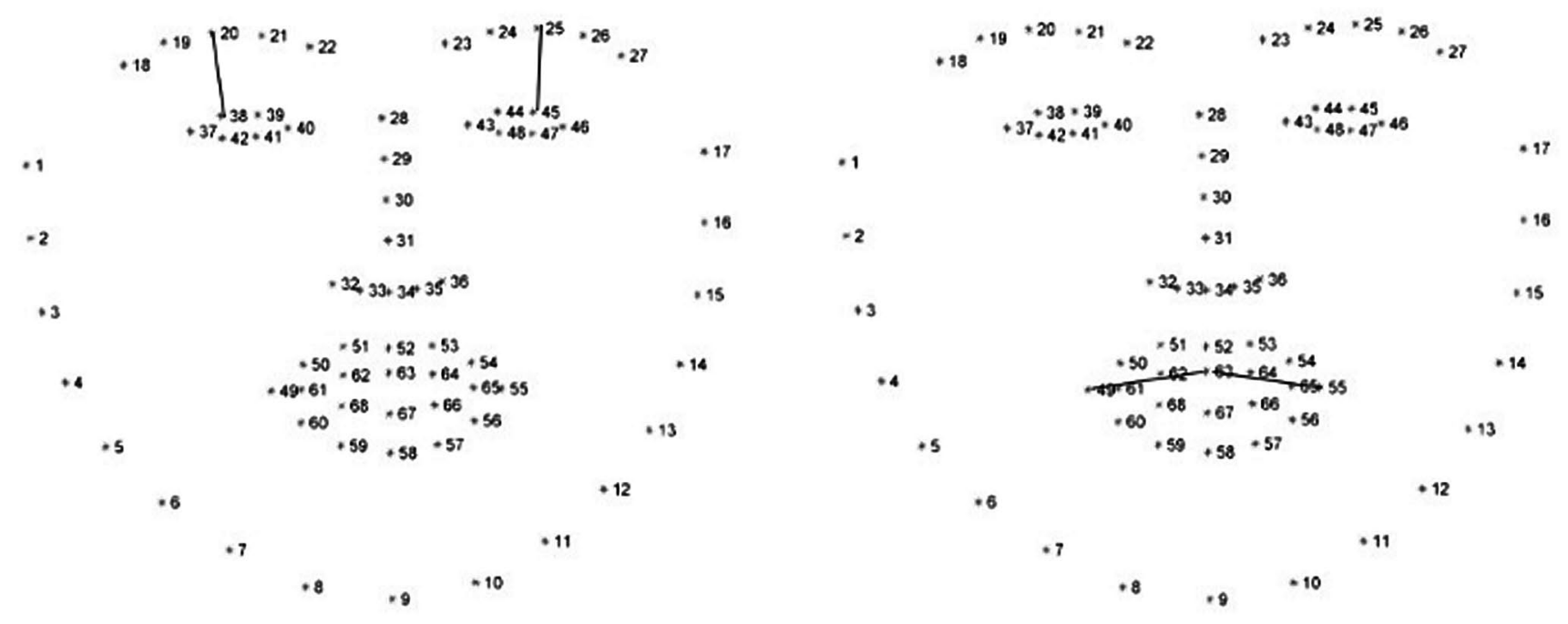

Fig. 2 Points to identify on the face the distances (continuous line) between eyebrows-upper eyelids (left) and the mouth corners (right) in the healthy and the affected side, individuated by "decisional trees" [19] 
significant. All computations were performed using $\mathrm{R}$ version 3.5.3 (2019-03-11) — “Great Truth" Copyright (C 2019 The R Foundation for statistical Computing and Graph Pad Prism vers. 6.01.

\section{Results}

The morphological curves of the two movements (forehead frowning and smiling) in both the normal and the UPFP situation derived from the two methods of analysis with and without reflective markers were comparable (Figs. 3, 4). The partial values of the shift differences of eyebrow elevation in the vertical plan when frowning the forehead ((shifts of the eyebrow from the eyelid) and in the horizontal plan when smiling (shift of the two mouth corners from the center of the upper lip) between the healthy and affected side for each subject belonging to HB grade II-V are shown in Table $1 \mathrm{a}$ and $\mathrm{b}$, respectively. The partial range specific for each $\mathrm{HB}$ grade of the shift differences was as follows:

- Eyebrow elevation was 0.40-2.86 (mean 1.65) in HBII; 3.76-4.46 (mean 4.03) in HBIII; 4.54-5.81 (mean 5.20) in HBIV; and 6.33-11.07 (mean 8.18) in HBV.

- Smiling was 0.32-2.75 (mean 1.34) in HBII; 3.00-3.90 (mean 3.37) in HBIII; 3.99- 4.65 (mean 4.25) in HBIV; and 4.99-6.24 (mean 5.56) in HBV.

The statistical comparison of the shift difference ranges in the different $\mathrm{HB}$ grades exhibited a significant difference among each HB grade $(p \leq 0.0001)$ both for the partial and total values. ANOVA tests showed that the mean difference during forehead frowning increased significantly as the HB grade increased ( $F=78.02 ; R^{2}=0.90$ (Fig. $6 \mathrm{a}$ ) and that the mean shift difference during smiling increased significantly

\section{Morphological patterns of contractions in the normal face with (1) and without markers (2)}

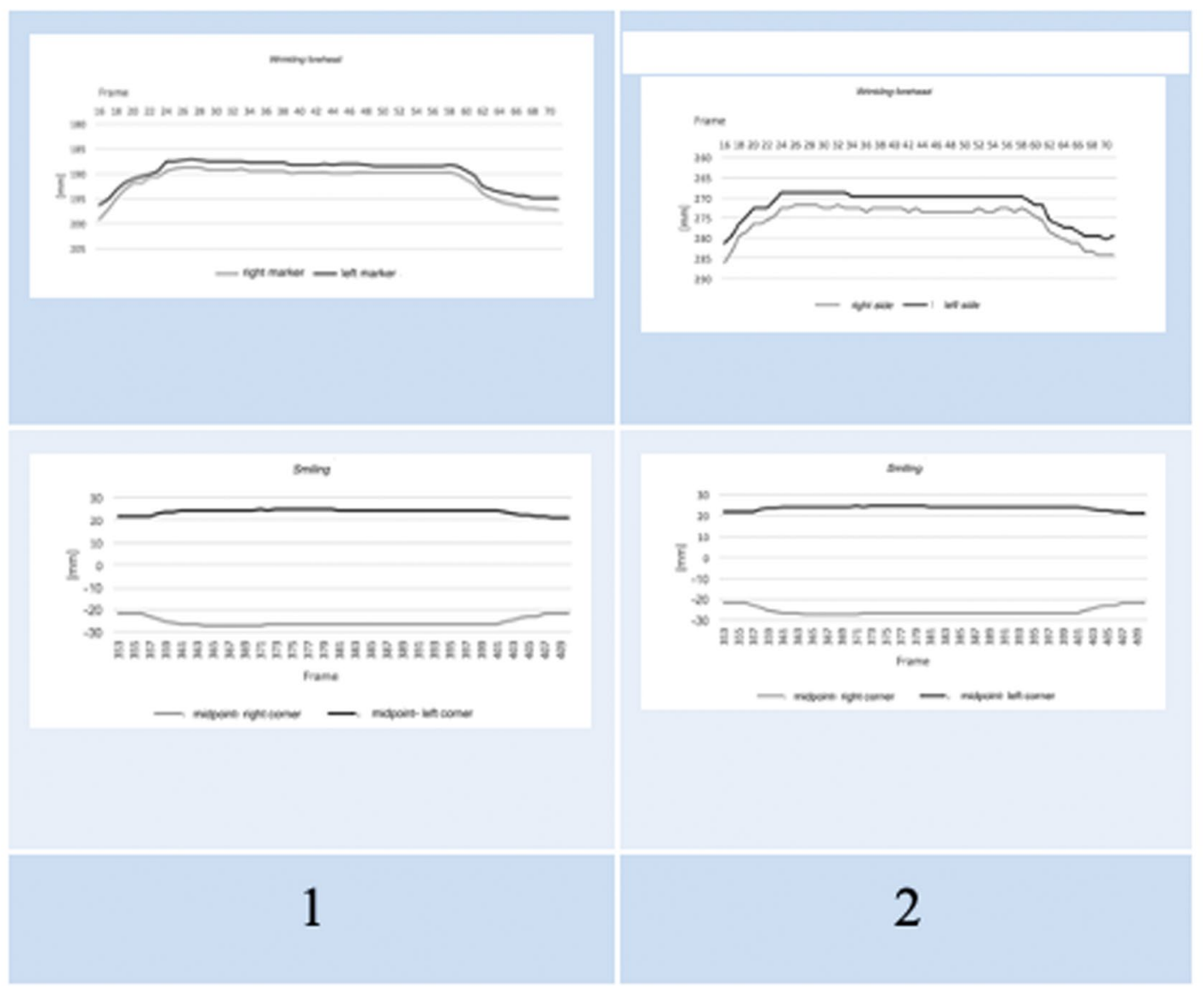

Fig. 3 Morphological comparison between marker [1] and markerless [2] point shifting in the normal face during forehead frowning and smiling 


\section{Morphological patterns of contractions in the UPFP grade HBV with (1) and without markers (2)}

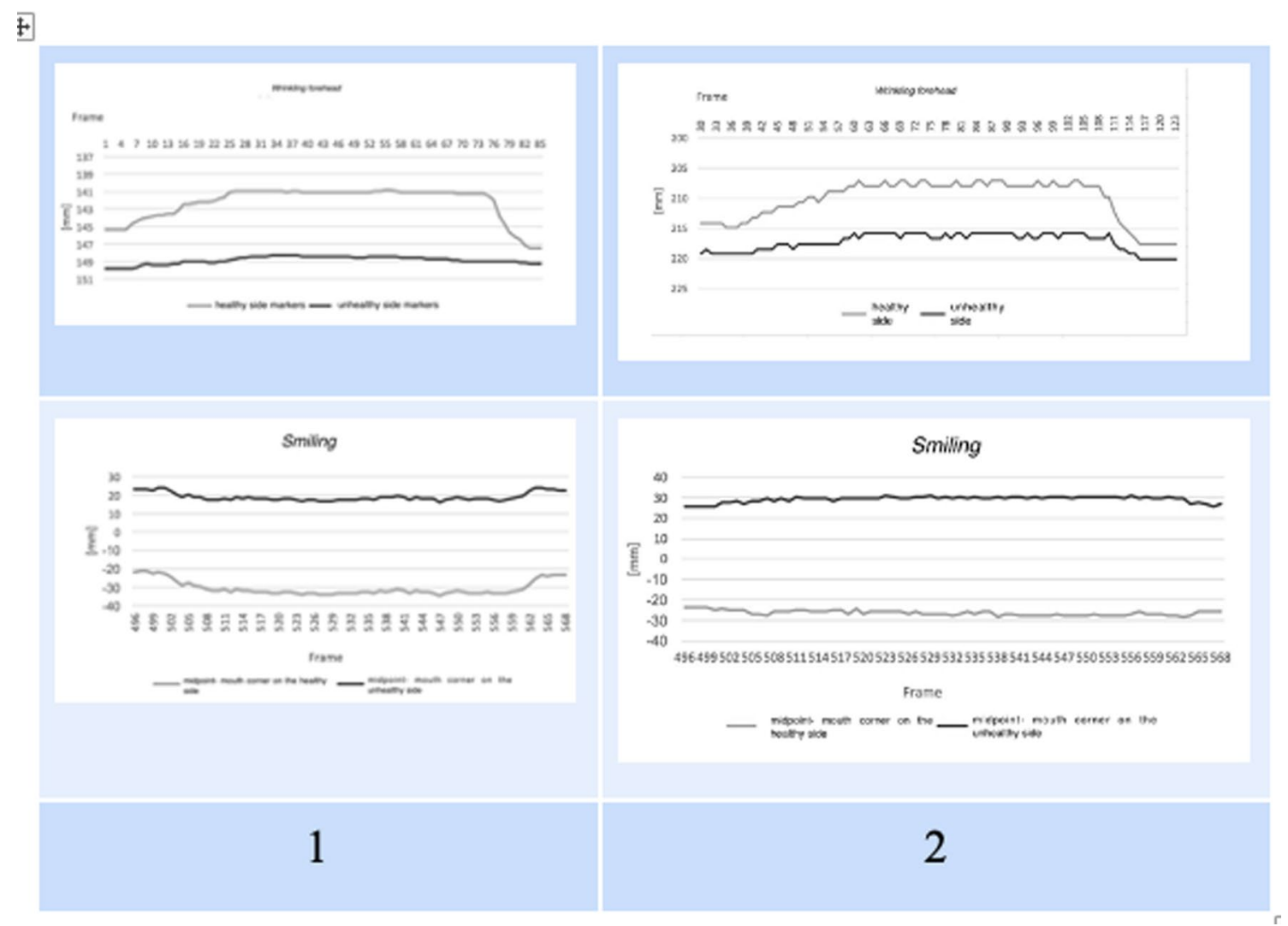

Fig. 4 Morphological comparison between marker [1] and markerless [2] point shifting in the paralyzed face of HBV grade patients, during forehead frowning and smiling

during smiling as the HB grade increased $(F=107.7$; $R^{2}=0.92$ ) (Fig. 6b).

The total scores of shift differences between the two face sides are summarized in Table 2 and have been 1.03-5.13 for HBII; 6.89-8.13 for HBIII; 8.98-10.35 for HBIV; and 11.49-16.39 for HBV.

The $p$ value for the total scores of the shift differences in the HB grades was significant $(<0.0001)$. For the total scores of the shift differences, the ANOVA test also showed a strict positive correlation with the HB grade increase (ANOVA test: $F=183.0 ; R^{2}=0.95$ ).

Regarding the shift ratio, the asymmetry indices for the frontal region were in the range of 0.74-0.98 (mean 0.85) in HBII; 0.54-0.68 (mean 0.61) in HBIII; 0.1-0.2 (mean 0.46) in HBIV; and 0.03-0.38 (mean 0.22) in HBV (Table 3a). In the mouth region, the ranges of shift ratio were 0.73-0.94 (mean 0.82) in HBII; 0.55-0.69 (mean 0.62) in HBIII; 0.43-0.51 (mean 0.48) in HBIV; and 0.1-0.34 (mean 0.24) in HBV (Table 3b). The ranges were statistically different for both the partial and the total values $(p=0.0001)$. The ANOVA test showed that the asymmetry index of the frontal $\left(F=96.68 ; R^{2}=0.91\right)$ and the mouth regions $(F=220$; $\left.R^{2}=0.96\right)$ decreased significantly as the HB grade increased (Fig. 5). A negative correlation between the mean total scores of the asymmetry index and the HB grade increase was observed $\left(F=207.50 ; R^{2}=0.96\right)$.

\section{Discussion}

The wide variety of available clinical and objective methods for the diagnosis of UPFP suggests their importance for providing reliable grading classifications for prognostic and therapeutic purposes. The traditional clinical classifications $[1,2]$ are mostly subjective and related to individual experience. Moreover, they present limitations for defining 
Table 1 a, b: Partial shift differences between healthy versus affected side in each HB grade during vertical (forehead frowning) and horizontal (smiling) face movements: the mean shift differences increase significantly with the HB grade increase $(p \leq 0.0001)$

Shift differences in the healthy and affected side during forehead frowning

\begin{tabular}{lllll}
\hline Score & HB II & HB III & HB IV & HB V \\
\hline Patient 1 & 0.87 & 3.89 & 5.56 & 6.93 \\
Patient 2 & 0.61 & 4.24 & 5 & 11.07
\end{tabular}

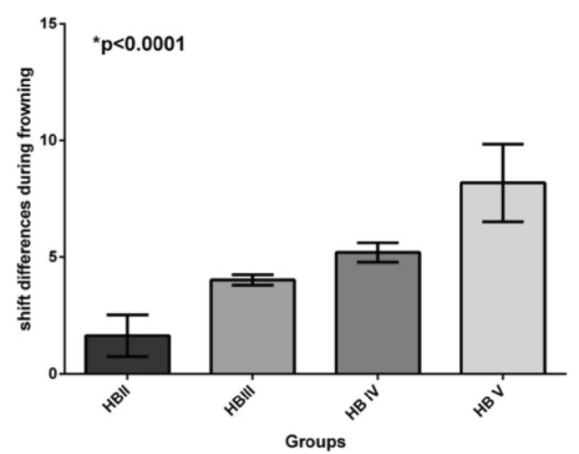

*Anova test: $F=78.02 ; R^{2}=0.90$

$\begin{array}{lllll}\text { Patient 3 } & 2.81 & 4 & 5.23 & 6.5 \\ \text { Patient 4 } & 1.66 & 3.8 & 4.54 & 6.32 \\ \text { Patient 5 } & 0.39 & 3.75 & 5.26 & 6.65 \\ \text { Patient 6 } & 2.53 & 3.95 & 4.82 & 7.60 \\ \text { Patient 7 } & 1.58 & 3.87 & 5.01 & 10.23 \\ \text { Patient } 8 & 2.86 & 4.23 & 4.96 & 8.65 \\ \text { Patient 9 } & 1.89 & 4.12 & 5.78 & 9.48 \\ \text { Patient 10 } & 1.26 & 4.45 & 5.80 & 8.32 \\ \text { Mean } & 1.65 & 4.03 & 5.20 & 8.18 \\ \text { Max } & 2.86 & 4.46 & 5.81 & 11.07 \\ \text { Min } & 0.40 & 3.76 & 4.54 & 6.33 \\ \text { St. dev. } & 0.89 & 0.23 & 0.42 & 1.67\end{array}$

Shift differences in the healthy and affected side during smiling

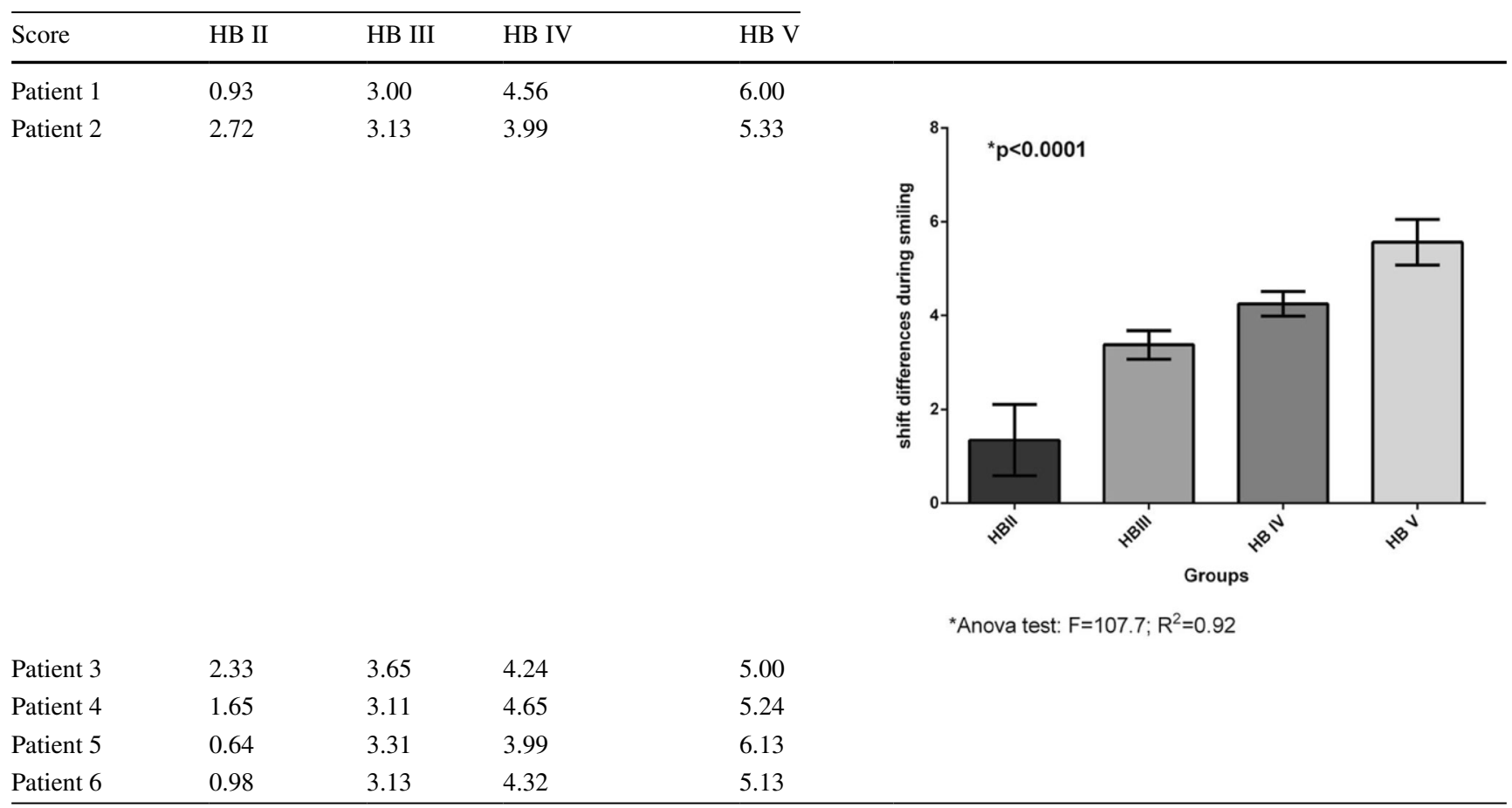


Table 1 (continued)

Shift differences in the healthy and affected side during smiling

\begin{tabular}{lllll}
\hline Score & HB II & HB III & HB IV & HB V \\
\hline Patient 7 & 1.33 & 3.57 & 4.04 & 4.99 \\
Patient 8 & 0.32 & 3.90 & 4.02 & 5.55 \\
Patient 9 & 0.82 & 3.69 & 4.56 & 5.99 \\
Patient 10 & 1.73 & 3.26 & 4.11 & 6.24 \\
Mean & 1.34 & 3.37 & 4.25 & 5.56 \\
Max & 2.72 & 3.90 & 4.65 & 6.24 \\
Min & 0.32 & 3.00 & 3.99 & 4.99 \\
St. Dev. & 0.76 & 0.30 & 0.26 & 0.48
\end{tabular}

Table 2 Absolute and mean values of the shift differences of the total facial movements in each HB grade

Total shift differences in the healthy and affected sides

\begin{tabular}{lllrr}
\hline Score & HB II & HB III & HB IV & HB V \\
\hline Patient 1 & 1.80 & 6.89 & 10.12 & 12.92 \\
Patient 2 & 3.3 & 7.36 & 8.98 & 16.39 \\
Patient 3 & 5.13 & 7.65 & 9.47 & 11.49 \\
Patient 4 & 3.31 & 6.91 & 9.19 & 11.56 \\
Patient 5 & 1.03 & 7.06 & 9.25 & 12.78 \\
Patient 6 & 3.51 & 7.08 & 9.14 & 12.73 \\
Patient 7 & 2.91 & 7.44 & 9.05 & 15.22 \\
Patient 8 & 3.18 & 8.13 & 8.98 & 14.20 \\
Patient 9 & 2.71 & 7.81 & 10.35 & 15.47 \\
Patient 10 & 2.98 & 7.71 & 9.91 & 14.56 \\
Mean & 2.99 & 7.40 & 9.44 & 13.73 \\
Max & 5.13 & 8.13 & 10.35 & 16.39 \\
Min & 1.03 & 6.89 & 8.98 & 11.49 \\
St. dev. & 1.07 & 0.41 & 0.50 & 1.68 \\
\hline
\end{tabular}

the mimic deviations of the face in quantitative terms. In particular, in the HBGS classification, the presence of synkineses starts to be included from Grade III on [1], whereas the SBGS assigns a specific partial score to synkineses that is masked by the final sum that includes the partial scores of the static and dynamic situations of the face [2]. The digital systems, mostly based on sophisticated software, do not provide a description of the facial alterations in all their aspects, both quantitatively and qualitatively, apart from being at times difficult to perform and time-consuming.

To date, an objective method that could assess all the qualitative modifications of the face mimic as hypercontraction or synkineses on both the affected and healthy side has not yet been proposed. Although none of the subjects included in the present study showed synkinesis in either side of the face, one may assume it useful to adopt an objective method to confirm the clinical classifications and monitor the sequels from a UPFP via clinical observations.

The objective methodologies proposed for the analysis of the face movement have been based on three main elements: video recording, systems for capturing the face structures under static and dynamic positions, and comparison of the affected and healthy side. Several studies have based their analysis on video recordings of the main face movements traced by reflector markers placed on several points of the face sectors $[4,5,15,19]$. Recently, one methodology has been validated by demonstrating the significant correlation between both HB grades and HB grades derived from SBGS classification, as well as $\mathrm{HB}$ grades derived from the marker analysis $[15,16]$. It is important to stress that this procedure may be rapid and easy, but also encompasses some difficulties when placing the markers due to individual physiognomic characteristics of the patient's face, such as an undefined eyebrow or the presence of a mustache.

A few markerless computing analyses studies have recently been reported [20-23], and three-dimensional techniques have been used to document the facial motions [10-14].

The present study has attempted to develop a markerless automatic system for the analysis of the face movements through the recognition of specific points for each structure of interest. The software was first trained on countless images of face movements of healthy subjects, using the "machine learning" method [17], with the recognition of 68 points of interest manually created via an "ensemble of tree decision" [18].

The strength of the present study is that the markerless analysis has been assessed on the video recordings of the subjects already classified and validated as HB grade in previous studies $[15,16]$, combining the objective marker analysis with two traditional clinical classification (HBGS and SBGS). 
Table 3 a-b: Partial shift ratio of forehead (a) and mouth (b) regions of all patients in each HB grade: the shift ratio decreases with the HB increase

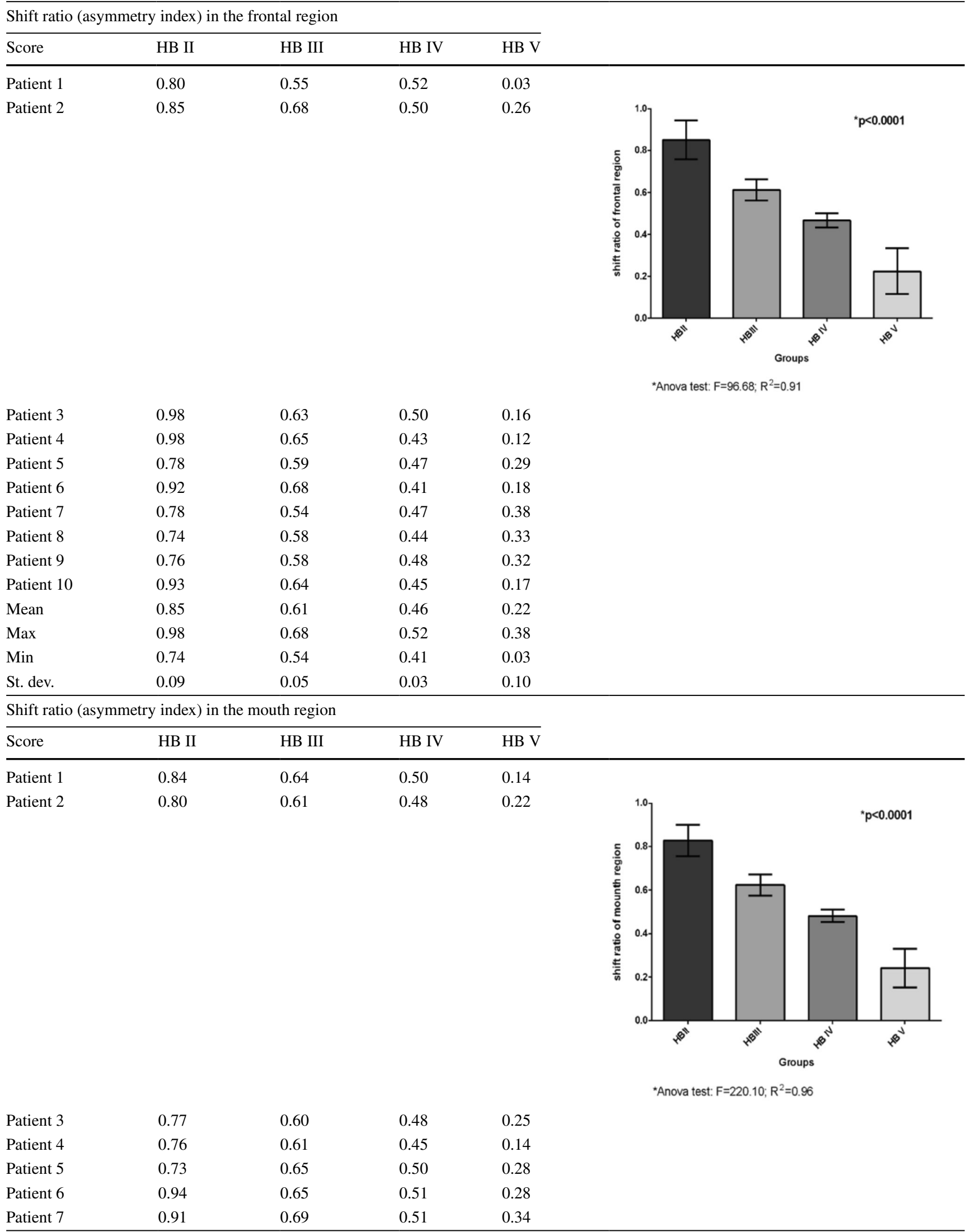


Table 3 (continued)

Shift ratio (asymmetry index) in the mouth region

\begin{tabular}{lllll}
\hline Score & HB II & HB III & HB IV & HB V \\
\hline Patient 8 & 0.78 & 0.55 & 0.50 & 0.33 \\
Patient 9 & 0.84 & 0.55 & 0.43 & 0.10 \\
Patient 10 & 0.91 & 0.68 & 0.45 & 0.33 \\
Mean & 0.82 & 0.62 & 0.48 & 0.24 \\
Max & 0.94 & 0.69 & 0.51 & 0.34 \\
Min & 0.73 & 0.55 & 0.43 & 0.10 \\
St. dev. & 0.07 & 0.04 & 0.02 & 0.08 \\
\hline
\end{tabular}

SHIFT RATIO IN THE TWO FACE MOVEMENTS

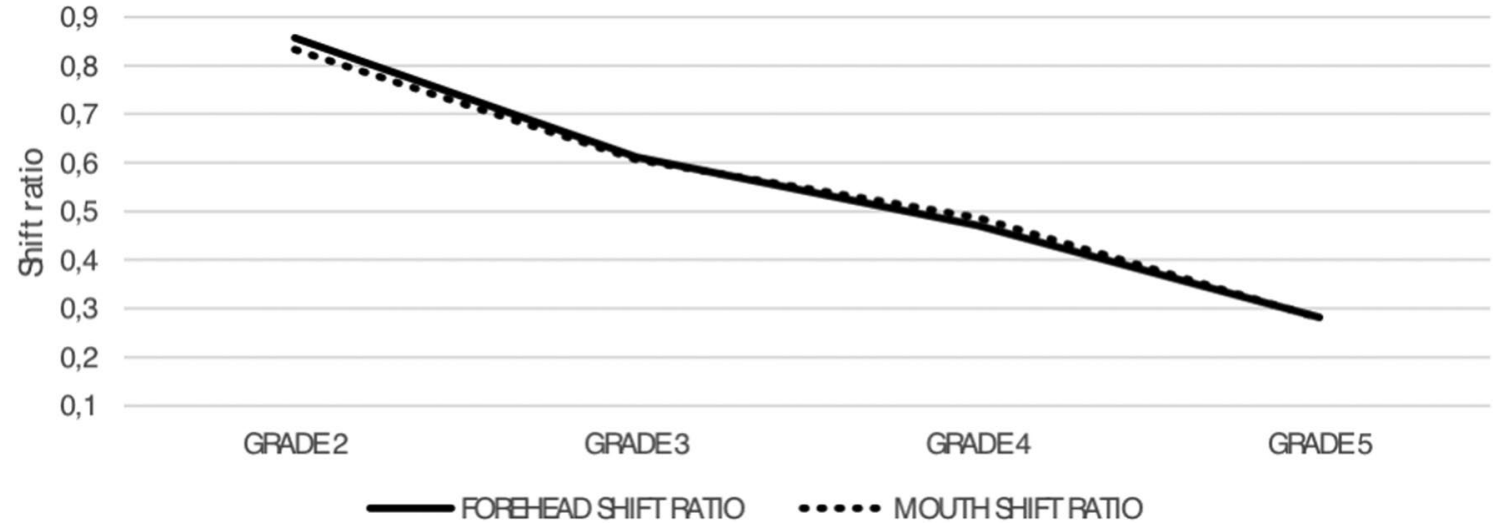

Fig. 5 The asymmetry index of the frontal and mouth regions decreases with the HB grade increase

A similar markerless study based on the software learning and tree decision has recently been reported [22]; however, photograms instead of videoclips were used, increasing the risk of not exactly quantifying the point distances during the face movements.

Among the possible merits of the present study it is worth stressing that, in addition to the differences of the distances between the two sides of the face, an asymmetry index between the two face sides for each movement, with scores specific for each HB grade, has been calculated. As a matter of fact, the shift differences between the two sides of the face exhibited range values that were significantly different and not overlapping among the HB grades in both movements. In addition, the partial and total ranges of the shift differences increased with a positive correlation with respect to the $\mathrm{HB}$ increase. Moreover, the range values for the asymmetry indices were significantly different and did not overlap among the HB grades, and the partial and total scores of the two movements were negatively correlated to the $\mathrm{HB}$ increase, as also indicated by the values from the normal subjects used for validating the previous study, having minimum and maximum values (0.99 and 1.2) greater than the other HB grades [16].

Based on these results, one may assume that the ranges of shift differences and asymmetry indices between the two face sides are sensible and specific for each HB grade considered.

A possible limitation of the present study is the fact that facial function was not evaluated via all possible face movements given that the assessment only evaluated two movements: the movement produced by forehead frowning, such as that noted in an expression of astonishment, and the movement produced when smiling. In particular, regarding the movement related to eye closure, it is known to be clinically important to separate minor (grade III and better) from severe cases (grade IV and worse). These movements were not analysed because it was not possible to define a sensible asymmetry index describing the palsy grade. In fact, it is likely to assume that it is not easy to modulate the entity of the contraction during eye closure, especially in subjects affected by a UPFP, as shown by variable asymmetry indices found in subjects with the 
same HB grade. This information gap, hence, needs to be bridged by combining the present objective procedure with direct evaluation of eye closure.

The methodology adopted in the present study seems to be valid due to the following reasons. The morphology of the markerless points considered to define the two movements in the affected and healthy sides corresponds to the marker method. Second, the methodology has been applied to videorecordings of subjects classified according to HBGS, HBGS derived from SBGS and HBGS derived from marker analysis [16]. Third, this methodology allowed us to identify, for each HB grade, specific ranges of shift differences between the two face sides and specific ranges of asymmetry index for each HB grade. These ranges were assessed for each movement, both individually and together.

In conclusion, the markerless objective method used in the present study may be useful to implement the conventional clinical classifications, given that it maintains the video information during the analysis time and can provide important data on those movements that the traditional subjective methods are unable to assess.

Funding Open access funding provided by Università degli Studi di Roma La Sapienza within the CRUI-CARE Agreement.

Open Access This article is licensed under a Creative Commons Attribution 4.0 International License, which permits use, sharing, adaptation, distribution and reproduction in any medium or format, as long as you give appropriate credit to the original author(s) and the source, provide a link to the Creative Commons licence, and indicate if changes were made. The images or other third party material in this article are included in the article's Creative Commons licence, unless indicated otherwise in a credit line to the material. If material is not included in the article's Creative Commons licence and your intended use is not permitted by statutory regulation or exceeds the permitted use, you will need to obtain permission directly from the copyright holder. To view a copy of this licence, visit http://creativecommons.org/licenses/by/4.0/.

\section{References}

1. House JW, Brackmann DE (1985) Facial nerve grading system. Otolaryngol Head Neck Surg 93(2):146-147

2. Neely SG, Cherian NG, Dickerson CB, Nedzelski JM (2010) Sunnybrook facial grading system: reliability and criteria for grading. Laryngoscope 120(5):1038-1045

3. Meier-Gallati V, Scriba H, Fisch U (1998) Objective scaling of facial nerve function based on area analysis (OSCAR). Otolaryngol Head Neck Surg 118:545-550

4. Linstrom CJ (2002) Objective facial motion analysis in patients with facial nerve dysfunction. Laryngoscope 112:1129-1147

5. Linstrom CJ, Silverman CA, Susman WM (2000) Facial-motion analysis with a video and computer system: a preliminary report. Am J Otol 21:123-129

6. O'Reilly BF, Soraghan JJ, McGrenary S, He S (2010) Objective method of assessing and presenting the House-Brackmann and regional grades of facial palsy by production of a facogram. OtolNeurotol 31:486-491
7. Katsumi S, Esaki S, Hattori K, Yamano K, Umezaki T, Murakami S (2015) Quantitative analysis of facial palsy using a three-dimensional facial motion measurement system. AurisNasus Larynx 42:275-283

8. Kecskés G, Jóri J, O’Reilly BF, Viharos L, Rovó L (2011) Clinical assessment of a new computerised objective method of measuring facial palsy. ClinOtolaryngol 36(4):313-319

9. Mitre EI, Lazarini PR, Dolci JE (2008) Objective method for facial motricity grading in healthy individuals and in patients with unilateral peripheral facial palsy. Am J Otolaryngol 29:51-57

10. Coulson SE, Croxson GR, Gilleard WL (1999) Three-dimensional quantification of "still" points during normal facial movement. Ann OtolRhinolLaryngol 108:265-268

11. Lin SC, Chiu HY, Ho CS, Su FC, Chou YL (2000) Comparison of two-dimensional and three-dimensional techniques for determination of facial motion-absolute movement in a local face frame. $\mathbf{J}$ Formos Med Assoc 99:393-401

12. Nakata S, Sato Y, Gunaratne P, Suzuki Y, Sugiura S, Nakashima S (2006) Quantification of facial motion for objective evaluation using a high-speed three-dimensional face measurement system-a pilot study. OtolNeurotol 27:1023-1029

13. Hartmann J, Meyer-Marcotty P, Benz M, Häusler G, StellzigEisenhauer A (2007) Reliability of a method for computing facial symmetry plane and degree of asymmetry based on 3d-data. J OrofacOrthop 68:477-490

14. Frey M, Giovanoli P, Gerber H, Slameczka M, Stüssi E (1999) Three-dimensional video analysis of facial movements: a new method to assess the quantity and quality of the smile. PlastReconstrSurg 104:2032-2039

15. Monini S, Marinozzi F, Atturo F, Bini F, Marchelletta S, Barbara M (2017) Proposal of a videorecording system for the assessment of Bell's Palsy: methodology and preliminary results. OtolNeurotol 38:1178-1185

16. Monini S, Filippi C, Marinozzi F, di Traglia M, Bini F, Marchelletta S, Ferraioli M, Margani V, Marinelli A, Barbara M (2019) Validation of the objective assessment of facial movement with a new software-based system. ActaOto-Laryngol 139:456-460

17. Guarin DL, Dusserdolp J, Hadlock TA (2018) A machine learning approach for automated facial measurements in facial palsy. JAMA Facial Palsy Surg 20:335-337

18. Kazemi V, Sullivan J (2014) One millisecond face alignment with an ensemble of regression trees. CVPR 2014:1-8

19. Somia NN, Rash GS, Epstein EE, Wachowiak M, Sundine MJ, Stremel RW, Barker JH, Gossman D (2000) A computer analysis of reflex eyelid motion in normal subjects and in facial neuropathy". ClinBiomech 15:766-771

20. Jorge JJ Jr, Pialarissi PR, Bors GC, Squella SAF, de Gouveia MF, Saragiotto JC Jr, Gonçalves VR (2012) Objective computerized evaluation of normal patterns of facial muscles contraction. Braz J Otorhinolaryngol 78:41-51

21. Al-Anezi T, Khambay B, Penguin MJ, O’Leary E, Ju X, Ayoub A (2013) New method for automatic tracking of facial landmarks in 3D motion captured images (4D) Int J Oral Maxillofac Surg 42:9-18.

22. Mothes O, Modersohn L, Volk GF, Kligerman C, Witte OW, Schlattmann P, Denzler J, Guntinas-Lichius O (2019) Automated objective and marker-free facial grading using photographs of patients with facial palsy. Eur Arch Oto-Rhino-Laryngol 276:3335-3343

23. Kim HS, Kim SY, Kim YH, Park KS (2015) A Smartphone-Based automatic diagnosis system for facial nerve palsy. Sensors (Basel) $15: 756-768$

Publisher's Note Springer Nature remains neutral with regard to jurisdictional claims in published maps and institutional affiliations. 